\title{
Design of Process Centrifugal Compressor: an Adapted
}

\section{Algorithm}

\author{
Muhammad Ali Ashjari ${ }^{1}$, Hassan Ali Marefat ${ }^{2}$ and Majid Reza Shahhosseini ${ }^{3}$ \\ 1. Department of Mechanical Engineering, Jolfa International Branch, Islamic Azad University, Jolfa 54417-34335, Iran \\ 2. Department of Mechanical and Aerospace Engineering, Science and Research Branch, Islamic Azad University, Semnan \\ 35135-179, Iran
}

3. Design Department, Oil Turbo-Compressor Equipment, Tehran 11548-38415, Iran

Received: August 27, 2013 / Accepted: October 14, 2013 / Published: February 28, 2014.

\begin{abstract}
Considering the essential and influential role of centrifugal compressors in a wide range of industries makes most of engineers research and study on design and optimization of centrifugal compressors. Centrifugal compressors are the key to part of oil, gas and petrochemical industries as well as gas pipeline transports. Since complete 3D design of the compressor consumes a considerable amount of time, most of active companies in the field, are profoundly interested in obtaining a design outline before taking any further steps in designing the entire machine. In this paper, a numerical algorithm, named ACDA (adapted compressor design algorithm) for fast and accurate preliminary design of centrifugal compressor is presented. The design procedure is obtained under real gas behavior, using an appropriate equation of state. Starting from impeller inlet, the procedure is continued on by resulting in numerical calculation for other sections including impeller exit, volute and exit diffuser. Clearly, in any step suitable correction factors are employed in order to conclude in precise numerical results. Finally, the achieved design result is compared with available reference data.
\end{abstract}

Key words: Process centrifugal compressor, adapted design algorithm, real gas.

\section{Introduction}

Centrifugal compressors have a wide range of applications in gas, oil, petrochemical, airplane, HVAC industries and so forth. Centrifugal compressor is invented and manufactured by Auguste Rateau late in 19th century. His single-stage prototype on his test-stand compresses $0.5 \mathrm{~m}^{3} / \mathrm{s}$ of atmospheric air to a discharge pressure of 1.5 bars at rotational speed of 20,000 rpm [1]. Such compressors have also been used in process industry since early 1900 that one of the earliest applications was as blast furnace blowers for steel mills [2].

Although the main components of centrifugal compressors do not differ significantly, there are

Corresponding author: Muhammad Ali Ashjari, assistance professor, research fields: computational fluid dynamic, dynamic simulation, and turbomachinery. E-mail: maliashjari@iauj.ac.ir. considerable improvements of overall polytropic efficiency and sizing in compressors offered by OEMs (original equipment manufacturers) today. Optimized design procedures along with improved manufacturing methods have been the most essential factor in the advancement of these days' high-performance centrifugal compressors. For a flow coefficient (dimensionless form of flow rate) greater than 0.08 , the general trend of peak compressor efficiency over the past 60 years is shown in Fig. 1.

Design in the industries means to size a compressor after the receipt of an order according to a philosophy with proven components. Fig. 1 shows that most of the companies have been working on 3D modeling and design of compressor over decades in order to improve efficiency. However, there is a great interest growing in the industry in $1 \mathrm{D}$ detail design recently. Since complete compressor sizing which is performed 


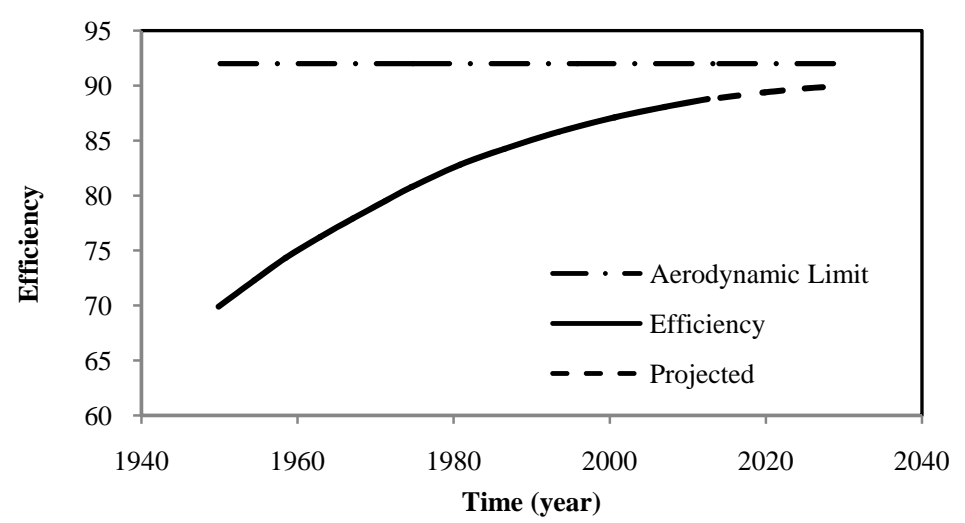

Fig. 1 Efficiency trend over the past 60 years [2].

through 3D sizing and in some cases dealing with CFD (computational fluid dynamics), costs a large portion of budget and time, most of companies keen on obtaining initial calculated design results.

Acquiring 1D numerical results could help industries to decide whether they should take another step ahead to run 3D sizing or not. Therefore, it is highly appreciated whether the output of one dimensional procedure would be accurate and precise enough to make decision based on themselves.

There are different approaches and methods in design of centrifugal compressors. In 1989, Cumpsty [3] published his book on compressor aerodynamics and provided basic equations and explanations for every individual section of different kinds of compressors. On the other hand, Aungier [4] published a book on centrifugal compressor and addressed his method and procedure for centrifugal compressor sizing, which can be used either for 3D or 1D modeling. In 2004, Lüdtke [5] dedicated a chapter of his book for a case study on detail design of centrifugal compressor. The book addresses aero-thermodynamic procedure which resulted in a geometric outcome that is checked by rotor-dynamic feasibility calculation for further reliability. Cumpsty mostly presented basic equations and descriptions on the matter, however, Aungier and Lüdtke provided a procedure in order to design compressor.

In this paper, a new approach is presented in order to bring all together in one organized and structured algorithm named ACDA (adapted compressor design algorithm). In fact, ACDA is a precise combination and optimization of previous design procedures in order to have a simple, accurate, and fast compressor design tool. The algorithm uses multiple programming loops along with numerical methods in order to result in an accurate and rigorous $1 \mathrm{D}$ design of centrifugal compressor. In this paper, a preliminary design calculation is presented in the first step. Most important obtained parameters are employed to run different sections of centrifugal compressor which cover impeller inlet, 1D impeller profile, impeller exit, volute and conical exit diffuser. Design procedure starts through calculation of overall values for entire compressor (zero dimensional analysis) and is continued by adding suitable correction factors so as to achieve detail design values. Every individual section's numerical results accuracy highly depends on previous section's output results. Therefore, whole sections' numerical results notably rely on initial thermodynamic input which includes real gas properties and pseudo-critical properties.

\section{Problem Definition}

As mentioned earlier, there are different approaches and procedures available in the literatures, however, no one constructs a straight and clear algorithm in order to code a computer application to design centrifugal compressor. Although someone can extract an algorithm through these literatures, it would consume a lot of time to do so. In this paper, an adapted algorithm namely ACDA, mostly based on Lüdtke's procedure in combination with other applied methods, is provided. 
Every part of mentioned procedure is provided in individual sections and all their complexities of turning their calculation procedure into a computer coding algorithm are described in detail. Along with descriptions, the flowcharts of different code loops are provided to clarify any misunderstanding of the procedure. In addition, the mentioned procedure is described and discussed by M.H. Ali et al. [6], and most of equations along with discussions are referred to in this work.

\section{Pre-design Calculations}

As presented in Marefat et al., before impeller inlet calculation is started, the pre-design section should be provided. In pre-design stage, suction volume flow, compressor total polytropic head, number of stages, maximum tip speed and maximum tip speed Mach number or machine Mach number [6] must be calculated. These parameters are so essential to be determined since they are of compressor characteristics. In addition, since inlet and outlet condition of compressor are known, therefore, based on them one can calculate pseudo-critical properties, such compressibility factor and isentropic volumetric exponent, at inlet and outlet of compressor. However, different suction and discharge sections of every individual stage should be calculated through numerical methods. ACDA provides an accurate method in order to determine value of pseudo-critical properties, mostly discussed in Ref. [6].

The error criteria used in this paper is 10-6, based on type of the used variables. In addition, in pseudo-critical properties of calculation procedure, it is necessary to use a suitable and efficient EOS (equation of state). In this paper, since the working fluid in compressor is hydrocarbon in order to achieve lowest deviation, LKP EOS [7] is employed to calculate mentioned properties. However, RKS EOS [8] is also can be used with an acceptable deviation for the considered working fluid.

Moreover, temperature at discharge condition
( $\left.T_{\text {disch }}\right)$ of a stage is calculated through the following equation:

$$
\begin{gathered}
T_{\text {disch }}=T_{\text {suc }} \times R P^{\text {pow }} \\
\text { pow }=\frac{K v_{m e} \times \eta_{p b}}{K v_{m e}-1}
\end{gathered}
$$

where, $K v_{m e}, \eta_{p b}$ and $R P$ mean isentropic volumetric exponent, basic polytropic efficiency and compressor pressure ratio, respectively. Furthermore, $Z m e$ and $K v_{m e}$ are average value of the parameters at suction and discharge sections of a stage.

\section{Impeller Inlet Algorithm}

Pre-design stage has a very influential role on entire procedure. The results concluded from this section are used as guideline throughout the procedure. In pre-design section, ACDA determines number of stages as well as pseudo-critical properties as mentioned earlier. The number of stages is needed since ACDA could understand that how many times every section's calculation must be carried out. ACDA implicates arrays in order to assign different stages' values of parameters. Therefore, different stages' pseudo-critical properties are used to calculate each stage's tip speed $\left(u_{2}\right)$, tip speed Mach number $\left(M a_{u 2}\right)$, blade inlet diameter $\left(d_{1}\right)$, blade inlet angle $\left(\beta_{1}\right)$ and impeller inlet width $\left(w_{1}\right)$ [5].

At impeller inlet section, the optimizing procedure of eye diameter/impeller diameter ratio $\left(d_{E} / d_{2}\right)$ should be presented in order to approach minimum impeller inlet losses. The minimizing procedure and conditions are addressed in detail in Ref. [6], however, ACDA optimizes impeller eye diameter through an algorithm, which has two nested loop. The inner loop (Fig. 2) calculates meridional speed $\left(c_{m 0}\right)$ at impeller eye section and the outer loop (Fig. 3) calculates actual static volume flow/total process volume flow ratio $\left(\dot{V}_{0^{\prime}} / \dot{V}_{0 t}\right)$ [6]. In this procedure, optimum eye diameter is desired to be accurately calculated, however, eye diameter is used inside inner loop to calculate meridional speed at impeller eye section.

Since impeller inlet area section and impeller eye area section are roughly the same the following 


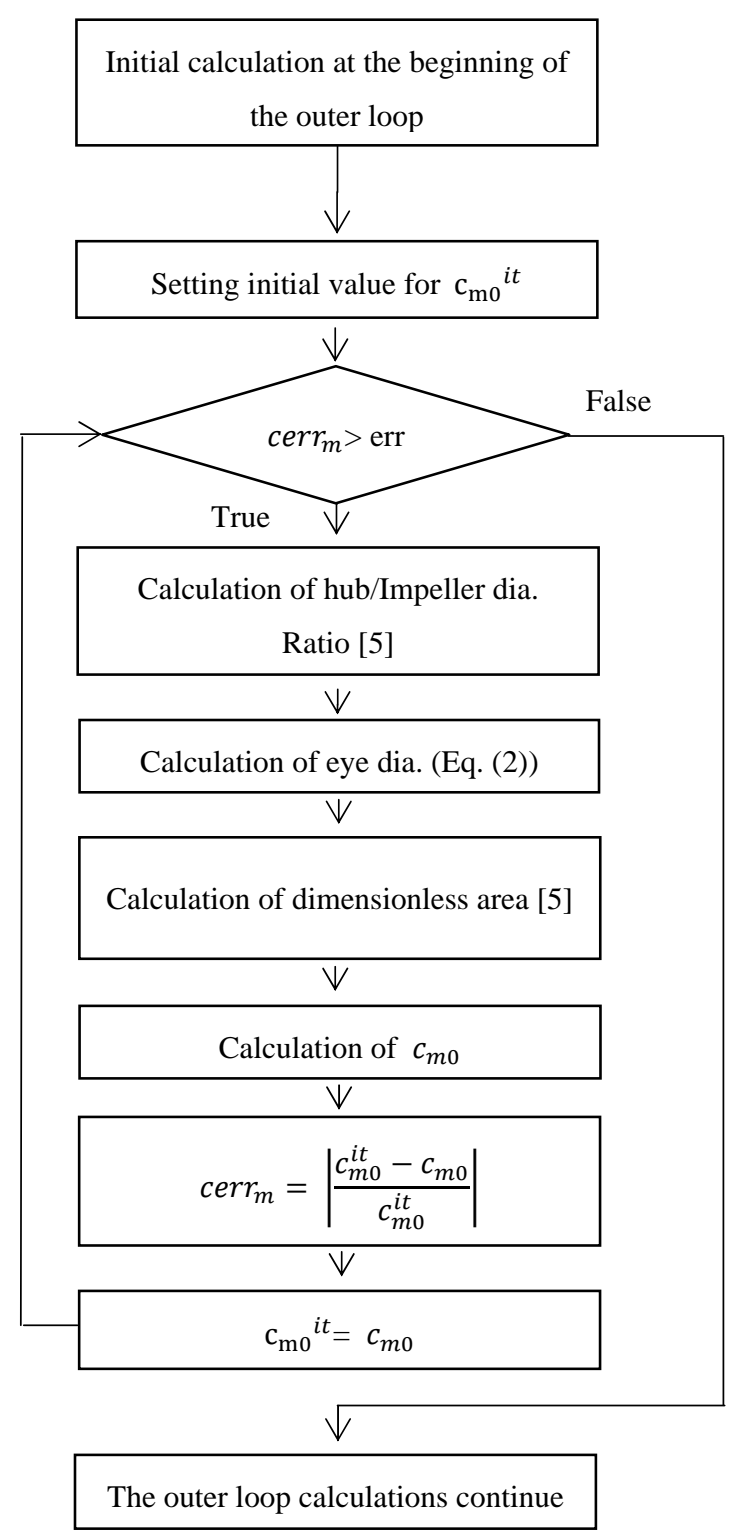

Fig. 2 Inner loop flowchart: Meridional speed calculation.

equation can be obtained in order to calculate impeller eye diameter:

$$
d_{E}=\sqrt{4 \dot{V}_{0^{\prime}} / \pi c_{m 0}+d_{H}{ }^{2}}
$$

where, $d_{H}$ is hub diameter which is considered as a input variable in the paper, in contrast with Lüdtke's procedure. And, $\dot{V}_{0^{\prime}}$ is actual static volume flow, which is calculated through some correction factors described by Marefat et al. [6]. In the literature, it is also recommended to consider impeller eye diameter some $5 \%$ smaller than optimum value to reduce

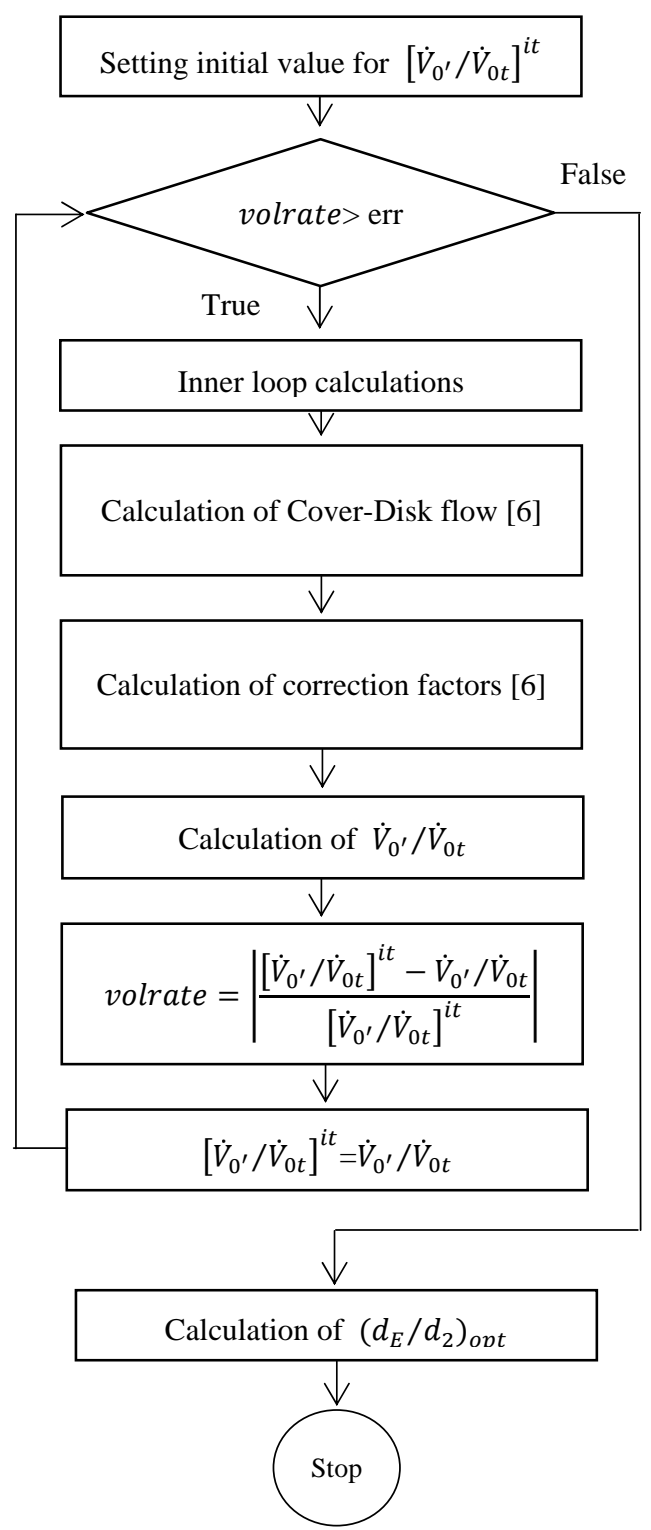

Fig. 3 Outer loop flowchart: $\dot{V}_{0^{\prime}} / \dot{V}_{0 t}$ calculation.

cover-disk stresses at the section. In addition, err is as the same as the mentioned one.

\section{Impeller Exit Algorithm}

The basis for calculation of impeller exit width $\left(b_{2}\right)$ is continuity equation. In order to obtain impeller exit width, inlet and exit flow coefficients $\left(\varphi, \varphi_{3}\right)$, reaction $(r)$ and work input factor (s) should be primarily calculated [6].

The calculation of impeller volume ratio $\left(\dot{V}_{3} / \dot{V}_{0 t}\right)$, results in calculation of impeller exit width using Eq. (3): 


$$
b_{2} \propto \varphi d_{2} \frac{\dot{V}_{3} / \dot{V}_{0 t}}{4 \varphi_{3}}
$$

Moreover, in order to calculate static parameters at diffuser's inlet, it is necessary to obtain impeller polytropic efficiency $\left(\eta_{p I}\right)$. Therefore, as a rule of thumb, one can calculate the parameter from corrected polytropic efficiency $\left(\eta_{p-c o r}\right)$ resulted by applying correction factors mentioned in impeller exit width flowchart [5]:

$$
\eta_{p I}=\sqrt{\eta_{p-c o r}}
$$

\section{Volute and Conical Exit Diffuser}

The aerodynamic design of the volute or scroll remains a rather qualitative and somewhat subjective process [4]. The quadratic internal volute design procedure performs through calculating any through-flow area at entire volute azimuth as per the continuity equation. Rearranging this correlation for volute section, Eq. (5) is obtained.

$$
A_{v o}=\frac{\dot{V}_{\vartheta}}{c_{u}}
$$

Volute consists of two main sections, namely curved and straight sections. The curved section starts with a quadratic cross section area, which becomes rectangular at a specified wrap angle. The beginning of rectangular section b (radial cross section width) is considered constant and a (axial cross section width) increment. By reducing volute effective area the maximum efficiency drops by less than $1 \%$ and as $\varphi$ increases the difference between full volute area curve and reduced volute area curve becomes more notable.

Each volute is followed by a conical exit diffuser. Since most of the exit diffusers in compressors have a rectangular inlet areas and circular exit area, the stall tends to occur at smaller area ratios. Pressure recovery factor is mostly affected by exit diffuser length and area ratio. According to Lüdtke [5] acceptable value for pressure recovery is more than 0.5 , which means area ratio more than 0.3 as well as length/diameter ratio more than 4.5. These values are preferable due to exit diffuser design which is secured not to go on stall.

\section{Results}

Obtained results from developed program are presented here. In this paper the case study considered in Ref. [5] is taken to be analyzed.

Using these input data, and employing mentioned procedure, the results in Tables 1 and 2 are obtained for different sections. Table 1 presents preliminary numerical results. These results are significantly essential for further numerical calculation, since their role is as guideline to make sure whether computation takes place precisely or not.

In Table 3 , the influence of disk diameter $\left(d_{20}\right)$ on polytropic efficiency of compressor is studied. It is clear that reduction in disk diameter improve the efficiency of compressor a little. On the other hand, having two different disk diameters for each individual stage can also improve the efficiency in the price of increment in impeller angular stresses. Therefore, it is advisable to keep disk diameter, and consequently, impeller exit diameter constant.

As mentioned above, although variable value for disk diameter of each stage can improve efficiency as slightly as reduction in constant disk diameter, it increases angular stress on impeller as well, which reduces compressor continues operation life-time. One can also study the influences of variant hub diameter on performance of compressor. In the following table the case is considered and numerical results are presented.

Obviously, decreasing hub diameter increases compressor polytropic efficiency marginally. Moreover, it must be notified that hub reduction can

Table 1 Preliminary numerical results.

\begin{tabular}{llcc}
\hline Item & Unit & Value & $\begin{array}{c}\text { Max. } \\
\text { value }\end{array}$ \\
\hline Number of stages & - & 2 & 4 \\
Tip speed of impeller & $\mathrm{m} / \mathrm{s}$ & 220.4 & 261.3 \\
Stage polytropic head & $\mathrm{kJ} / \mathrm{kg}$ & 26.9 & - \\
Polytropic head & $\mathrm{kJ} / \mathrm{kg}$ & 53.9 & - \\
Polytropic efficiency (basic) & $\%$ & 82.54 & - \\
Flow coefficient & - & 0.11 & - \\
Mach number, tip speed & - & 0.52 & 1.08 \\
Impeller diameter & $\mathrm{m}$ & 0.30 & - \\
Discharge temperature & ${ }^{\circ} \mathrm{C}$ & 74.57 & - \\
\hline
\end{tabular}


Table 2 Gas pseudo-critical properties.

\begin{tabular}{lllll}
\hline \multirow{2}{*}{ Parameter } & \multicolumn{2}{l}{ Design } & \multicolumn{3}{l}{$\begin{array}{l}\text { Ref. Data } \\
\text { (Lüdtke, 2004) }\end{array}$} \\
\cline { 2 - 5 } & St. 1 & St. 2 & St. 1 & St. 2 \\
\hline Suc. comp. fac. & 0.88 & 0.90 & 0.883 & 0.889 \\
Disc. comp. fac. & 0.90 & 0.91 & 0.889 & 0.897 \\
Suc. Isen. Vol. Exp. & 1.37 & 1.37 & 1.33 & 1.35 \\
Disc. Isen. Vol. Exp. & 1.37 & 1.38 & 1.35 & 1.38 \\
Suc. Isen. Temp. Exp. & 1.32 & 1.30 & 1.31 & 1.31 \\
Disc. Isen. Temp. Exp. & 1.30 & 1.29 & 1.31 & 1.30 \\
\hline
\end{tabular}

Table 3 Disk diameter influence on compressor performance.

\begin{tabular}{lll}
\hline Case & $d_{20}(\mathrm{~mm})$ & $\eta_{p}(\%)$ \\
\hline Constant dia. & 318 & 84.16 \\
Constant dia. & 316 & 84.28 \\
Variable dia. & Stage 1 = 318 & 84.23 \\
\hline
\end{tabular}

affect rotational speed of impeller considerably. It is reasonable practice to optimize hub diameter considering efficiency in one side and rotational speed and angular stresses subsequently in the other side. Diffuser through path growth can augment compressor whole efficiency. Table 4 shade light on the discussion, and make it clear which increasing diffuser exit diameter/impeller exit diameter ratio $\left(d_{4} / d_{2}\right)$ is directly proportional to polytropic efficiency. Although it is economical to increase diffuser path, it is not applicable for most of the times due to void space absence.

Through Tables 3 and 4, it can be understood that $d_{4} / d_{2}$ affect compressor polytropic efficiency notably. It is true since diffuser is in charge of conversion of high kinetic energy exiting from impeller to static pressure. Therefore, longer diffuser has more time to do so rather than smaller diffuser.

In this paper, constant disk and hub diameter with maximum $d_{4} / d_{2}$ is considered, and the numerical results for impeller inlet and exit are presented as Table 5.

For the specified compressor detail design calculation results for volute and conical exit diffuser are presented in Table 6 and Figs. 4a and 4b.

The following conclusions would be achieved from the current work:

(1) The ACDA algorithm shows an acceptable deviation from the reference available data for the specific compressor. Since it uses robust and fast numerical algorithms, as mentioned earlier in the paper, its obtained results are reliable as the Tables 5 and 6 present;

(2) The procedure also has accurate convergence on the essential parameters such as eye diameter, hub and disk diameters which have direct effects on polytropic efficiency of compressor as shown in tables 3 and 4;

(3) All in all, the ACDA algorithm brings together effective procedure presented by Lüdtke et al [5] and reliable numerical algorithms to provide a fast and accurate zero-dimensional of centrifugal compressor working under different ambient conditions as well as various working fluids.

Table 4 Diffuser diameter influence on compressor performance.

\begin{tabular}{llll}
\hline Disk dia $(\mathrm{mm})$ & Hub dia $(\mathrm{mm})$ & $d_{4} / d_{2}$ & $\eta_{p}(\%)$ \\
\hline 318 & 105 & 1.3 & 77.9155 \\
318 & 105 & 1.6 & 83.3938 \\
318 & 105 & 1.8 & 84.1634 \\
\hline
\end{tabular}

Table 5 Impeller inlet \& exit numerical results.

\begin{tabular}{lllll}
\hline \multirow{2}{*}{ Parameter } & $\begin{array}{l}\text { Design } \\
\text { St. 1 }\end{array}$ & St. 2 & St. 1 & St. 2 \\
\hline Impeller diameter & 304 & 302 & 304 & 302 \\
Tip speed & 222.39 & 220.9 & 222.3 & 220.9 \\
Inlet flow Coeff.(ref $d_{2}$ ) & 0.104 & 0.0938 & 0.10 & 0.09 \\
Static/process vol. flow & 1.03 & 1.0315 & 1.04 & 1.03 \\
Opt. eye dia. ratio & 0.64 & 0.6481 & 0.66 & 0.65 \\
Rel. imp. in. width & 0.14 & 0.1296 & 0.13 & 0.12 \\
Rel. imp. ex. Width & 0.07 & 0.071 & 0.076 & 0.06 \\
Exit flow coeff. & 0.32 & 0.30 & 0.314 & 0.310 \\
Work in factor & 0.59 & 0.60 & 0.604 & 0.607 \\
Reaction & 0.614 & 0.617 & 0.616 & 0.617 \\
\hline
\end{tabular}

Table 6 Volute and exit diffuser numerical results.

\begin{tabular}{llll}
\hline \multirow{2}{*}{ Parameter } & \multirow{2}{*}{ Unit } & Design & $\begin{array}{c}\text { Ref. data, } \\
\text { (Lüdtke, 2004) }\end{array}$ \\
\cline { 3 - 4 } & & St. 2 & St. 2 \\
\hline Exit volume flow & $\mathrm{m}^{3} / \mathrm{s}$ & 1.32 & 1.25 \\
Slip/work input fac. ratio & - & 0.97 & 0.98 \\
Max. axial width & $\mathrm{mm}$ & 108.4 & 105.9 \\
Inlet dimension of exit diff. & $\mathrm{mm}^{2}$ & 18,740 & $19,909.2$ \\
Area ratio & - & 4.02 & 3.55 \\
Exit diff. length & $\mathrm{mm}$ & 70.8 & 81.8 \\
Pressure recovery coeff. & - & 0.50 & 0.51 \\
\hline
\end{tabular}




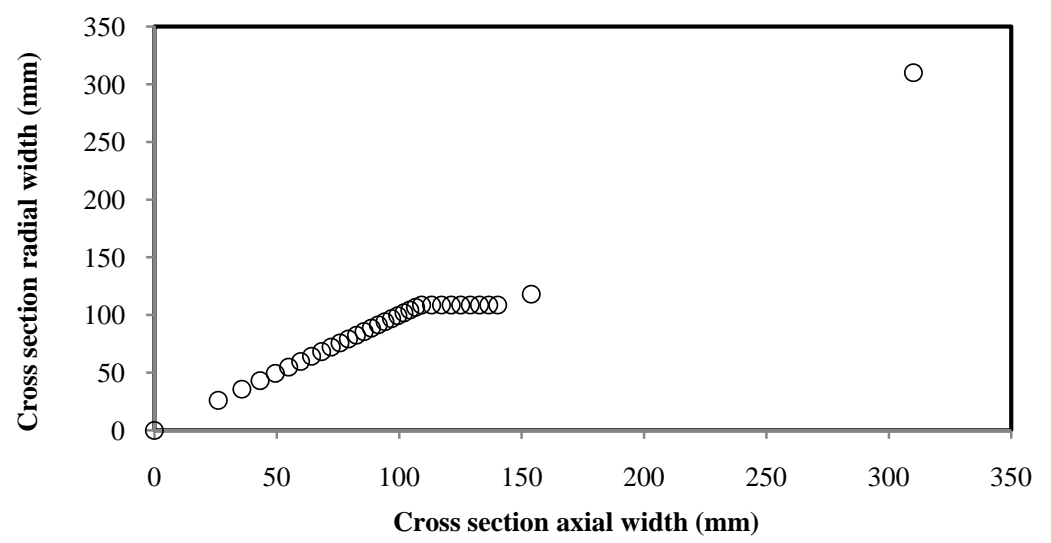

(a)

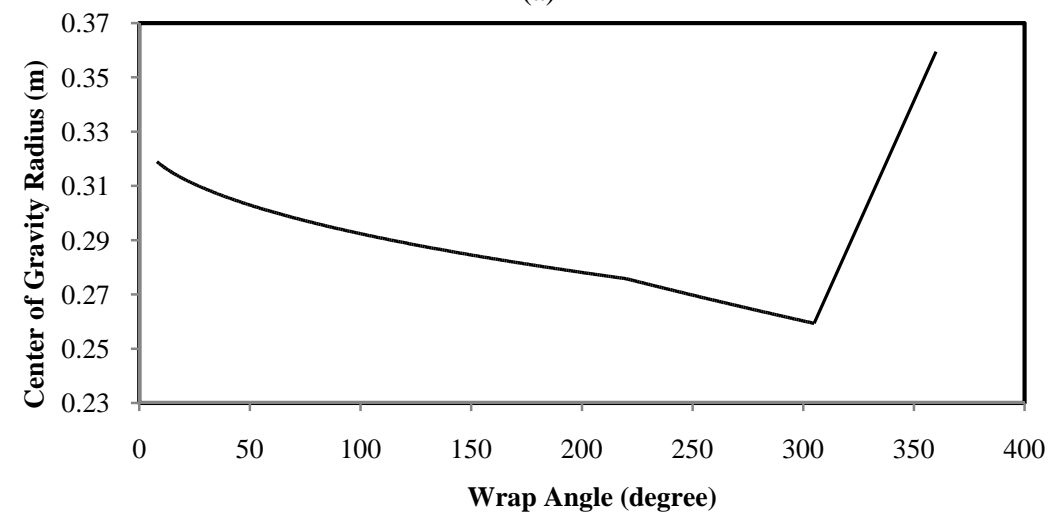

(b)

Fig. 4 (a) Volute and conical exit diffuser cross-section area; (b) center of gravity radius in different volute and conical exit diffuser azimuths.

\section{References}

[1] A. Engeda, From the crystal palace to the pump room, in: International Gas Turbine \& Aeroengine Congress, Stockholm, Sweden, 1998.

[2] M. Sorokes James, J. Kuzdzal Mark, Centrifugal compressor evolution, in 39th Turbo-machinery Symposium, USA, 2010.

[3] N.A. Cumpsty, Compressor Aerodynamics, Krieger Pub Co., 1989.

[4] R.H. Aungier, Centrifugal Compressors: A Strategy for Aerodynamic Design and Analysis, ASME Press, New York, 2000.
[5] H.K. Lüdtke, Process Centrifugal Compressor: Basics, Function, Operation, Design and Application, 1st ed., Springer, Berlin Heidelberg, 2004.

[6] M.H. Ali, M.R. Shahhosseini, M.A. Ashjari, Adapted design of process multi-stage centrifugal compressor and comparison with available data, International Journal of Materials, Mechanics and Manufacturing 1 (2) (2013) 195-199.

[7] B.I. Lee, M.G. Kesler, A generalized thermodynamic correlation based on three-parameter corresponding states, AICHE Journal 21 (3) (1975) 510-527.

[8] O. Redlich, J.N.S. Kwong, On the thermodynamics of solutions, Chemical Review 44 (1) (1949) 233-244. 